\title{
Comment on "Concerning the generation of geomagnetic giant pulsations by drift-bounce resonance ring current instabilities" by K.-H. Glassmeier et al., Ann. Geophysicae, 17, 338-350, (1999)
}

\author{
I. R. Mann ${ }^{1}$, G. Chisham ${ }^{2}$ \\ ${ }^{1}$ Department of Physics, University of York, York, UK \\ ${ }^{2}$ British Antarctic Survey, Natural Environment Research Council, Cambridge, UK
}

Received: 17 May 1999 / Accepted: 5 October 1999

Key words: Magnetospheric physics (energetic particles, trapped; MHD waves and instabilities) - Space plasma physics (wave-particle interactions)

\section{Introduction}

In their recent paper, Glassmeier et al. (1999) described observations of a giant pulsation $(\mathrm{Pg})$ measured by the Scandinavian magnetometer array. Using co-incident energetic proton observations made by GEOS-2 at a location nearly conjugate to their ground measurements, the authors identified a possible bump-on-tail at $\sim 67 \mathrm{keV}$. Using the azimuthal wave number and the period of the wave as derived from the ground-based magnetometer observations, Glassmeier et al. (1999) tried to test the hypothesis that the Pg they observed on the ground was driven by this bump-on-tail distribution through an unstable drift-bounce resonance (e.g., Southwood et al., 1969; Southwood, 1976). In order to be able to match their observations with theory, Glassmeier et al. (1999) derived a new resonance condition and claimed that at times when the conjugate ionospheres had asymmetric conductivity the usual integer- $N$ drift-bounce resonance condition could be satisified by a non-integer value $n$. We show in this comment that these calculations and this assertion are fundamentally flawed.

The standard drift-bounce resonance condition is written as

$\omega-m \Omega_{D}=N \Omega_{B}$ where $\omega$ and $m$ are the wave frequency and azimuthal wave number, $\Omega_{B}$ is the particle bounce frequency, $\Omega_{D}$ is the particle's bounce-averaged drift frequency, and $N$ is an integer (Southwood et al., 1969). For the Pg event observed by Glassmeier et al. (1999), the resonance condition was not satisfied for the observed $\omega$ and $m$, assuming a proton energy of $67 \mathrm{keV}$. To circumvent this problem, and to attempt to provide a causal link between the GEOS-2 particle signature and the $\mathrm{Pg}$ observed on the ground, Glassmeir et al. (1999) suggested a more general drift-bounce resonance condition than that derived by Southwood (1976) and given in our Eq. (1). They argued that if an asymmetry in the conjugate ionospheric conductivities exists, then it is possible for the resonance condition to be generalised to:

$\omega-m \Omega_{D}=n \Omega_{B}$

where $n \in \mathscr{R}$, i.e. any real number, $n$ being determined by the resonant particle path length between mirror points and the asymmetry in ionospheric conductivity.

Glassmeier et al. (1999) claimed that replacing integer $N$ with real $n$ "is a proper generalisation of the Southwood (1976) condition". If true, this represents a significant result since observers wishing to explain observations of ULF pulsations believed to be driven by drift-bounce resonance would be free to invoke noninteger values of $n$ into the resonance condition.

The mathematical formulation of Glassmeier et al. (1999) produces a resonance condition which infers that particles in drift-bounce resonance experience a time-independent continual increase in energy regardless of the value of $n$. We show that the calculations of Glassmeier et al. (1999) are in error and that the correct treatment retains the condition that $N$ be an integer. Only in the special drift-resonance case where $N=0$ does the particle experience a time-independent increase in energy along its path. The introduction of asymmetric ionospheric conductivities at the conjugate points in opposite hemispheres does not alter this conclusion. 


\section{Resonances on field lines with asymmetric ionospheric conductivities}

Glassmeier et al. (1999) considered the rate of change of particle energy due to interaction with a ULF wave, given by

$\delta \dot{W}_{B}(s)=q E_{\phi}(s) v_{D}(s) \exp [\mathrm{i}(m \phi-\omega t)]$,

where $q$ is the electric charge of the particle, $E_{\phi}(s)$ is the arc-length-dependent wave azimuthal electric field, $v_{D}(s)$ the arc-length-dependent particle azimuthal drift velocity, and $\phi$ is the azimuthal angle. Following the analysis of Glassmeier et al. (1999) we can replace $v_{D}(s)$ with its bounce averaged value $\left\langle v_{D}(s)\right\rangle=v_{D}$ and set the drift phase to $\phi=\Omega_{D} t$. If the electric field $E(s)$ is written as

$E(s)=\sum_{N=-\infty}^{\infty} A_{N} \exp \mathrm{i} N \Omega_{B} t$

as in Southwood (1976), then integrating the resulting expression for $\delta \dot{W}_{B}$ with respect to time [cf. Eq. (16) of Southwood, 1976] gives

$\delta W_{B}=q v_{D} \sum_{N=-\infty}^{\infty} A_{N} \frac{\exp \left[\mathrm{i}\left(m \Omega_{D}-\omega+N \Omega_{B}\right) t\right]}{\left(\omega-m \Omega_{D}-N \Omega_{B}\right)}$.

The dominant term in this summation is the resonant one for which $N$ satisfies the condition $\omega-m \Omega_{D}=N \Omega_{B}$ (cf. Eq. 1).

Glassmeier et al. (1999) argue that it is the expansion in Eq. (4) which forces $N$ to be an integer in Eq. (1). They claim that if the arc length position $s$ of the particle on the field line is instead approximated by a triangular function (see Glassmeier et al.'s Eq. (16) and the correction in their reply, Glassmeier, 1999) then the resulting expression for $\delta \dot{W}_{B}$ can be integrated without recourse to an expansion like Eq. (4).

Glassmeier et al. (1999) choose to write their electric field as

$E_{\phi}(s)=-\mathrm{i} E_{0} \exp \left[\mathrm{i} \frac{\alpha \pi s}{L}\right]$

where $L$ is the field line length, and where they claim that $\alpha$ can account for wave asymmetry about the equator. This allows them to generate the equation

$\delta \dot{W}_{B}=-\mathrm{i} E_{0} v_{D} \exp \left[\mathrm{i}\left(m \Omega_{D}-\omega\right) t+\mathrm{i} \frac{\alpha \pi s}{L}\right]$.

Using their triangular function to relate $s$ to $t$, Glassmeier et al. (1999) then integrate their expression for $\delta \dot{W}_{B}$ over one bounce cycle to give

$$
\begin{aligned}
\delta W_{B} \approx & -\mathrm{i} q E_{0} v_{D} \times\left\{\int_{0}^{T_{B} / 2} \exp \left[\mathrm{i}\left(m \Omega_{D}+n \Omega_{B}-\omega\right) t\right] \mathrm{d} t\right. \\
& \left.+\int_{T_{B} / 2}^{T_{B}} \exp \left[\mathrm{i}\left(m \Omega_{D}-n \Omega_{B}-\omega\right) t\right] \cdot \exp (\mathrm{i} n) \mathrm{d} t\right\}
\end{aligned}
$$

where $T_{B}=2 \pi / \Omega_{B}$ and $n$ is specified by $n=\alpha l / L$, where $l$ is the distance between the particle mirror points. They argue that the sum of these integrals will maximise if either $\omega-m \Omega_{D}-n \Omega_{B}=0$ (the first integral dominates), or $\omega-m \Omega_{D}+n \Omega_{B}=0$ (the second integral dominates), so that the generalised form of the resonance condition would be $\omega-m \Omega_{D}-n \Omega_{B}=0$, with $n \in \mathscr{R}$ and either positive or negative.

This mathematical treatment is flawed because of Glassmeier et al.'s (1999) incorrect treatment of the form of the wave electric field (stated in Eq. 6). An alternative and correct treatment can be considered by adopting an electric field of the form

$E_{\phi}(s)=E_{0}(s) \operatorname{expi} \psi(s)$,

see, e.g., Allan (1982). This formalism can describe the general form of the electric field eigenmodes supported by dipolar field lines with footpoints in conjugate hemispheres of asymmetric ionospheric conductivity (e.g. Allan and Knox, 1979a, b). Here $E_{\phi}(s)$ describes the time-independent amplitude variation of the electric field along the field line and $\psi(s)$ describes the fieldaligned phase.

For example, a fundamental (half-wavelength) harmonic with conjugately symmetric infinite ionospheric conductivities has $\psi(s)=0$ along the entire field line, and the wave represents an in-phase purely symmetric standing mode. When realistic finite conductivities are introduced the wave develops a small propagating component which can carry Poynting flux to the dissipative ionosphere. However, for realistic conductivities, the mode is still dominantly a standing mode along the majority of the field line; only very close to the ionosphere where the standing mode electric field is nodal does $\psi(s)$ become non-zero (see, e.g., Fig. 4 of Allan and Knox, 1979b, which shows a case with conjugately symmetric ionospheric conductivity of $\Sigma_{P}=10$ mhos). Even when the conductivities are made asymmetric (e.g., Fig. 5 of Allan and Knox, 1979b where $\Sigma_{P}=10,3$ mhos), non-critically damped modes retain the feature that $\psi(s) \approx 0$ along the vast majority of the field line, although in this case $E_{0}(s)$ is of course asymmetric.

The equation used by Glassmeier et al. (1999) (reproduced as Eq. 6 above) to describe the wave electric field, however, produces a phase which increases proportional to $s$ along the entire field line. Under Glassmeier et al.'s (1999) triangle approximation relating $s$ to $t$ this generates a field aligned phase for the resonant particle which is proportional to $t$ for all time. In fact, as shown by Allan and Knox $(1979 a, b)$ far from being proportional to $s$, the phase $\psi(s)$ remains approximately constant along almost the entire field line, except for the $180^{\circ}$ step phase changes which occur across the (near-) nodes of the eigenmodes.

Glassmeier et al.'s (1999) erroneous form of $E_{\phi}(s)$ leads to an incorrect linear relationship between fieldaligned phase and $t$, and it is this which causes them to infer that regardless of the value of $n$ a resonance condition can be generated in which the electric field in the frame of the particle is time-independent. This is incorrect, and the assertion by Glassmeier et al. (1999) that a non-integer $n$ can generate a viable drift-bounce resonance condition when the wave fields are asymmet- 
ric is wrong. When the correct analysis is undertaken it becomes clear that $N$ must be an integer for a genuine resonance to occur, and that in general the particles do not experience a time-independent electric field, except for the special case when $N=0$. The existence of this flaw can be clearly shown with a simple graphical analysis and we demonstrate this in detail.

\section{Graphical treatment of drift-bounce resonance}

Southwood and Kivelson (1982) developed a powerful graphical means of understanding the energy exchange between mirroring energetic particles and high- $m$ ULF waves. By mapping the path of the mirroring energetic particle in the wave rest frame, i.e. a frame which moves with the waves azimuthal phase speed, the possible conditions for drift-bounce resonance with different harmonic waves can be analysed. For example, Southwood and Kivelson (1982) show that purely symmetric (odd mode) waves may be driven through drift $(N=0)$, or drift-bounce $(N= \pm 2, \pm 4, \ldots)$ resonances, the $N=0$ resonance usually being dominant (Southwodd, 1976). Similarly, purely antisymmetric (even mode) waves may be excited by $N= \pm 1, \pm 3, \ldots$ drift-bounce resonances ( $N= \pm 1$ usually dominant).

In their paper, Glassmeier et al. (1999) considered the possibility of drift-bounce resonance driving asymmetric ULF wave modes whose line of symmetry/anti-symmetry is displaced from the equatorial plane. As discussed already, waves of this type are expected to be supported by field lines with asymmetric ionospheric conductivities at the conjugate points in opposite hemispheres (e.g. Allan and Knox, 1979a, b). Glassmeier et al. (1999) correctly concluded that in this case both asymmetric odd (with symmetry about a line displaced from the equatorial plane) and even (with anti-symmetry about a line displaced from the equator) mode waves might be driven at the same time by either even- or odd- $N$ resonances. In the asymmetric wave case the symmetries of the waves and particles are different. This means that there are some trajectories which involved no net transfer of energy in the symmetric case but in the asymmetric case can result in a secular decrease in particle energy. This in itself represents a very important result. However, it is the claim by Glassmeier et al. (1999) that these energy exchanges could be generated by non-integer- $n$ resonances which is in error.

To illustrate why this is the case, we can examine the physics of the resonance condition (1) as was described previously by Southwood and Kivelson (1982). In the frame of the wave, the particle's azimuthal drift speed is Doppler shifted by the azimuthal phase speed of the ULF wave $(\omega / m)$ so that in the wave frame $\dot{\phi}=\Omega_{D}-\omega / m$. For the case of an $N=0$ resonance, the wave and the particle move with the same azimuthal phase speed so that $\dot{\phi}=0$ and the particle "sees" a constant time-independent electric field. For other resonances, where both $N$ and hence $\dot{\phi}$ are $\neq 0$, the particles move with respect to the wave. In this case, the path of the particles must be examined carefully to

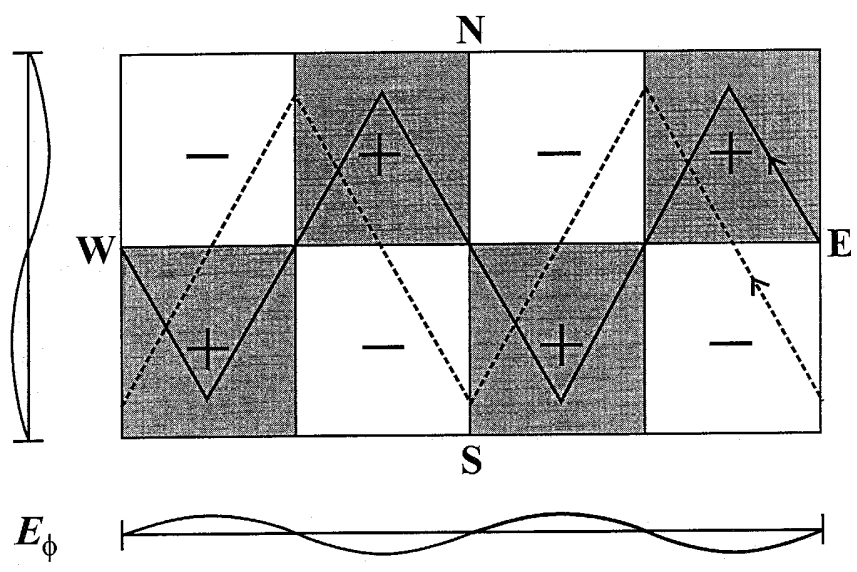

Fig. 1. Trajectories of two ions in the wave rest frame (solid and dashed lines) which are in $N=-1$ drift-bounce resonance with a second field aligned harmonic wave (after Southwood and Kivelson, 1982). The positive and negative signs represent the direction of wave electric field and the position of maximum amplitude

determine whether a particular wave harmonic can be resonant with a given particle trajectory.

In order for a particle to maintain any possible resonance and give energy to the waves, it must not have an energy loss over part of its trajectory totally cancelled out by subsequent energy gain later. This means that the particle must return to the same phase relative to the wave after an integer number of bounces in the wave frame. If the particle does not return to the same relative phase, its phase shifts with respect to the wave, the result being that no resonances and hence no sustained wave growth are possible. Mathematically, this is equivalent to requiring that the particles travel across an integer number $N$ of azimuthal wavelengths $\left(\lambda_{\phi}=2 \pi / m\right)$ in a bounce cycle. For example, equating the time for the particle to cross one wave azimuthal wavelength $\left(\lambda_{\phi} / \dot{\phi}\right)$ with the bounce time $2 \pi / \Omega_{B}$, gives the relation $m \dot{\phi}=\Omega_{B}$, i.e.,

$\omega-m \Omega_{D}=-\Omega_{B}$,

which is the same expression as Eq. (1) with $N=-1$. The situation is exactly analogous to the well-known wave particle cyclotron resonances where $\omega-k_{\|} v_{\|}=$ $N \Omega_{c}$. For cyclotron resonance, the Doppler shifted wave frequency must match an integer number $(N)$ of cyclotron frequencies $\Omega_{c}$.

The situation for drift-bounce resonance with $N=-1$ is schematically illustrated in Fig. 1 (adapted from Southwood and Kivelson, 1982), which shows two possible particle trajectories at different drift phases in the field of an antisymmetric (second harmonic) wave in the wave's rest frame. The trajectories shown are linear approximations to the particle bounce motion between mirror points, the same approximation as the triangular function adopted by Glassmeier et al. (1999) (their Eq. 16; see also the correction in their reply Glassmeier, 1999). On the dashed trajectory, an ion experiences equal positive and negative azimuthal electric fields over its path. In linear theory, where the action of the wave on the particle is considered over unperturbed paths, 


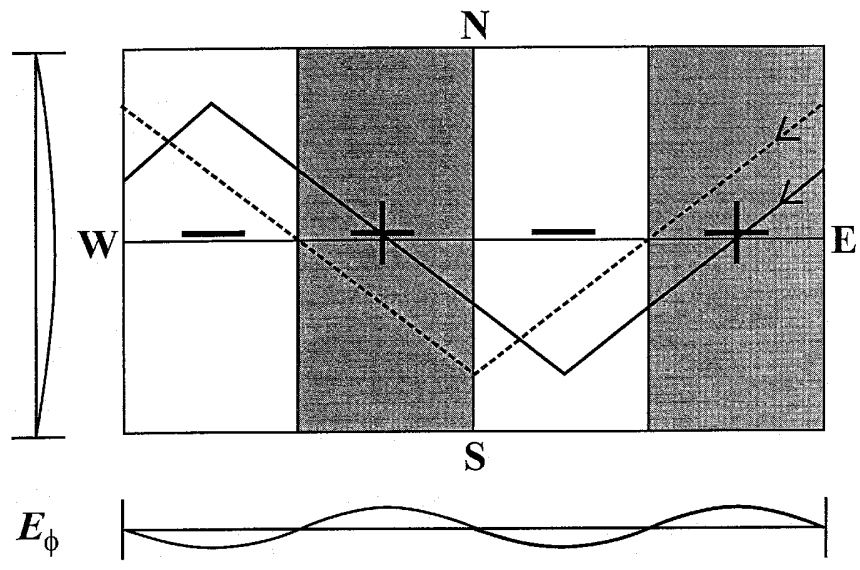

Fig. 2. Trajectories of two ions in $N=-2$ drift-bounce resonance with a fundamental field aligned mode (same format as Fig. 1)

the particle has zero net energy change. The solid line trajectory, however, shows an ion experiencing a positive azimuthal electric field over the whole of its path. Consequently, the ion is in resonance with the wave, and experiences a secular deceleration imparting its energy to the wave. If the local particle distributions are energetically favourable so that overall more particles are decelerated than accelerated then there is a net transfer of energy from the particles to the wave.

In Fig. 2 we show the situation for the $N=-2$ resonance with a symmetric fundamental mode wave. The dashed trajectory shows a particle crossing equal positive and negative azimuthal electric field regions and hence experiencing zero net (linear) energy change. The solid trajectory, however, crosses the equatorial plane at the times of maximum positive wave amplitude and reaches its mirror point at the times of maximum negative amplitude. Since the wave is a fundamental field-aligned harmonic, the electric field at the equator is greater than at the mirror points, the result being that the particle experiences a net (linear) deceleration over it's path. Again, under conditions where the particles have energetically favourable distribution functions energy can be transferred from the particles to the wave.

We can also consider the situation for non-integer- $n$. In particular we will demonstrate how it is impossible for the $n=0.4$ interaction, which Glassmeier et al. (1999) proposed as the driver of their Pg, to result in sustained wave growth. First, in Fig. 3, we consider a possible $n=0.4$ interaction between three particles of different drift phase with a perfectly symmetric odd mode wave (in this case the fundamental). Here $n=0.4$ represents the situation whereby, in the frame of the wave, during 5 bounce cycles the particles drift east through 2 azimuthal wavelengths. This means that $-2(2 \pi / m \dot{\phi})=5\left(2 \pi / \Omega_{B}\right)$, which gives $m \dot{\phi}=-2 \Omega_{B} / 5$ or alternatively that

$\omega-m \Omega_{D}=0.4 \Omega_{B}$.

Both the dashed paths (trajectories 1 and 3 ) in Fig. 3 traverse equal positive and negative field regions and hence there is no net (linear) deceleration. In a way similar to the $N=-2$ case shown in Fig. 2, however, the solid trajectory in Fig. 3 involves decelerations and accelerations of the particle in the positive and negative electric field regions which are not precisely symmetric. Indeed, although the particle crosses the equatorial plane in both positive and negative fields, the equatorial (maximum field-aligned amplitude) negative fields are encountered when the wave has maximum (temporal) amplitude. At times earlier and later than this, the particle moves away from the temporal maximum and towards the mirror points where the electric fields and hence the acceleration will be weaker. Conversely, there are two equatorial crossings in the positive $E_{\phi}$ regions close to, but on either side of, the temporal wave maxima which will cause particle deceleration. Due to the differences between the field aligned and azimuthal field variations, there is the hypothetical possibility for a small imbalance to occur between the positive and negative $E_{\phi}$ regions sampled on this trajectory. However, because the particles are repeatedly accelerated and decelerated any net energy exchange is likely to be insignificant. In particular, in the real situation, a particle on this trajectory will be affected non-linearly by the wave field accelerations/decelerations. This means that the precise phase of the particle trajectory will be shifted slightly over time so that any slight net deceleration over one set of five bounce cycles is likely to be phase shifted into an overall acceleration over the following set of cycles so that the effect tends to be cancelled. In this way we would expect the particles to experience phase mixing with respect to the waves, and hence there should be no overall energy transfer from the particles to the waves (this is not to be confused with the oscillations of waves at the local Alfvén eigenfrequencies whereby the phase of the waves with respect to each other increases in time, which has also been described as phase mixing, see, e.g., Mann and Wright, 1995).

This non-integer- $n$ phase mixing does not occur in integer- $N$ cases. For example, for the $N=-2$ case shown in Fig. 2, it can be seen that small non-linear perturbations to the particle trajectory maintain the resonance and allow for a secular net energy transfer

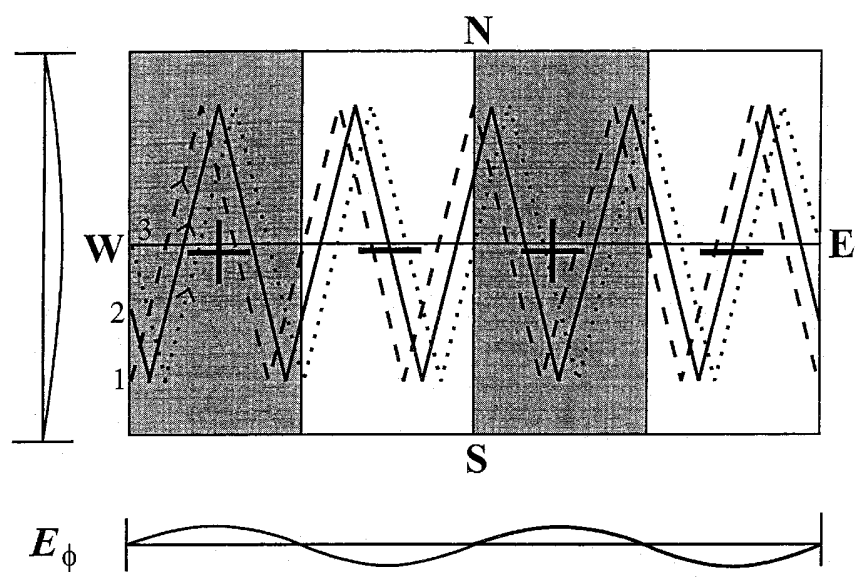

Fig. 3. Trajectories of three ions of different drift phase in an $n=0.4$ drift-bounce wave-particle interaction with a symmetric fundamental field-aligned harmonic (same format as Fig. 1) 


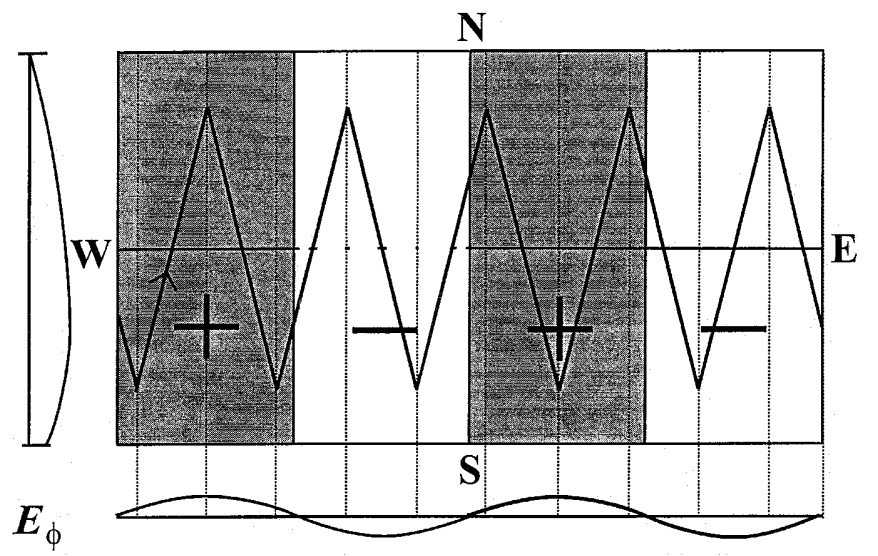

Fig. 4. Trajectory of an ion in an $n=0.4$ drift-bounce wave-particle interaction with an asymmetric fundamental field-aligned harmonic mode (same format as Fig. 1)

from the particle to the wave. In other words the noninteger- $n$ drift-bounce interactions, such as $n=0.4$, cannot be described as resonances and hence they are not viable candidates for driving ULF pulsations.

Glassmeier et al. (1999) claimed that an $n=0.4$ resonance might still be viable, however, if the interaction were with an asymmetric fundamental mode wave whose axis of symmetry is displaced away from the equatorial plane. In Fig. 4, we show this case, with the drift phase taken to be the same as the solid path (trajectory 2) from Fig. 3. The vertical dotted lines highlight the positions in wave phase where the particles reach their mirror points, and hence approximate the regions where for the Southern Hemisphere the particles would experience close to the maximum electric field magnitudes. Examining the trajectory carefully shows that whilst over some sections of the trajectory there appears to be the possibility for the particles to be strongly declerated by being closer to the wave amplitude maxima south of the equatorial plane, later in the orbit these effects are cancelled by the parts of the orbit which are closer to the northern mirror point where the electric field is weaker, so that the benefit is lost. As in the symmetric wave case shown in Fig. 3, there is the hypothetical possibility for a small imbalance between the linear acceleration and deceleration experienced over a trajectory of five particle bounce cycles, however, any imbalance is likely to be insignificant. Moreover, nonlinear orbit phase mixing removes the possibility of any overall energy exchange, so that even when the wave fields are asymmetric about the equator non-integer- $n$ interactions are not viable candidates for driving high- $m$ waves. This being the case, an alternative drift-bounce resonance with integer $N$ must be invoked if this is the mechanism responsible for driving the Pgs reported by Glassmeier et al. (1999).

\section{Alternative interpretation of data for integer- $N$ resonances}

Glassmeier et al. (1999) make the assumption that their Pg occurs as a consequence of drift-bounce resonance with energetic protons, and that an enhancement observed in the proton distribution function at $\sim 67$ $\mathrm{keV}$ offers a likely energy source. Although the correlation of the wave intensity with the proton enhancement in the 59-75 keV band appears quite convincing in Fig. 5 of Glassmeier et al. (1999), these protons fail to satisfy the drift-bounce resonance condition for integer $N$. Since drift-bounce resonance is a likely source of instability, we have made an estimate of the energy of protons which could lead to a resonance if an integer $N$ was assumed.

In our calculations we use the wave characteristics as observed on the ground ( $T \sim 100 \mathrm{~s} ; m \sim-26)$ and for the drift frequency $\Omega_{D}$ we use the value as defined by Chisham (1996) which includes both an energy dependent gradient-curvature term and electric field dependent convection and corotation terms. We assume that the $L$-shell of resonance is $L \sim 5.44$ (the dipole field $L$-shell of MUO, the station where maximum amplitude was observed), that the local time of the event can be expressed as $\phi \sim 135^{\circ}$ (i.e., $\sim 0900 \mathrm{MLT}$ ), that the pitch angle of the protons $\alpha \sim 20^{\circ}$, and that the convection electric field can be estimated by its dependence on $K_{p}$; in this case $K_{p}=4-$. Based on this, we estimate that the drift-bounce resonance condition is satisfied for energies $W \sim 12 \mathrm{keV} \quad(N=+1)$ and $W \sim 250 \mathrm{keV}$ $(N=0)$.

Protons of these energies will only contribute to wave growth if the particle distribution function $f$ is increasing with $W$ at these energies, i.e.

$\frac{\mathrm{d} f}{\mathrm{~d} W}=\frac{\partial f}{\partial W}+\frac{\mathrm{d} L}{\mathrm{~d} W} \frac{\partial f}{\partial L}>0$

This equation shows that instability can occur if there is a sufficient spatial gradient in some part of the resonant distribution (i.e. $\partial f / \partial L$ is large) or if the distribution is inverted at some point (bump-on-tail) so that $\partial f / \partial W>0$ (see Southwood et al., 1969). If we assume that a bump-on-tail distribution is responsible for the instability then we should be looking for a positive slope in the proton distribution function at either $W \sim 12 \mathrm{keV}$ or $W \sim 250 \mathrm{keV}$. The proton instrument used by Glassmeier et al. (1999) had an energy range from 28$402 \mathrm{keV}$ and so would not detect a bump-on-tail at lower energies. No bump-on-tail is observed at $250 \mathrm{keV}$ but this could be a result of the energy resolution of the instrument; only 10 energy channels exist between 28 and $402 \mathrm{keV}$.

We cannot be sure, without further evidence, if either of these particle populations is responsible for the growth of the Pg. However, the spacecraft data appear to suggest that the $\mathrm{Pg}$ is a fundamental mode wave which suggests that the $N=0$ solution $(W \sim 250 \mathrm{keV})$ may be the most likely. Particles of this energy have drift periods $\sim 1 \mathrm{~h}$ and so could have originated from the substorm injection observed $\sim 0530-0600$ UT. However, if protons with energies $\sim 67 \mathrm{keV}$ are to be implicated in the $\mathrm{Pg}$ generation then an alternative generation scenario to drift-bounce resonance needs to be found. 


\section{Summary}

Whilst the conclusion of Glassmeier et al. (1999) that both odd and even asymmetric wave modes could be driven by the same drift-bounce resonance is correct, we have shown that Glassmeier et al.'s (1999) subsequent assertion that non-integer- $n$ drift-bounce resonances could drive this type of asymmetric wave is in error. The ability for odd- $N$ resonances to drive both even and odd mode waves at the same time, so long as they are excited on field lines with conjugately asymmetric conductivies, may be very important. Indeed this might provide an explanation for the driving mechanism of some of the high- $m$ pulsations which have been previously reported in the literature. For example, Allan et al. (1983) reported observations of multiple harmonic high- $m$ pulsations which they believed could have been driven by drift-bounce resonance. However, because both even and odd modes were observed at the same time, the authors were forced to propose that both drift and bounce resonances (each resonant with very different parts of the energetic particle spectrum) were operating at the same time and in the same location. As Allan et al. (1983) point out, this is "an extremely complicated situation". If the possibility of some wave asymmetry is included then it could be possible for both even and odd modes to be driven by the same $N$ resonance.

Similarly, in a study of compressional high- $m$ waves, Takahashi et al. (1987) pointed out that whilst an $N= \pm 1$ drift-bounce resonance could have excited waves with the period and azimuthal wave number observed, their observations were of fundamental mode waves which (if symmetric) could not be excited by an $N= \pm 1$ drift-bounce resonance. However, if an ionospheric conductivity asymmetry were present then fundamental (albeit asymmetric) mode waves could be excited by $N= \pm 1$ drift-bounce resonance. We reiterate, however, that even when asymmetric modes are excited, it is only the integer- $N$ drift-bounce resonances which can exchange energy efficiently enough to give sustained wave growth via the well-known condition given in Eq. (1).

Acknowledgement. I.R.M. is supported by a UK PPARC Fellowship.

\section{References}

Allan, W., Phase variation of ULF pulsations along the geomagnetic field-line, Planet. Space Sci., 30, 339, 1982.

Allan, W., and F. B. Knox, A dipole field model for axisymmetric Alfvén waves with finite ionospheric conductivities, Planet. Space Sci., 27, 79, 1979a.

Allan, W., and F. B. Knox, The effect of finite ionospheric conductivities on axisymmetric toroidal Alfvén wave resonances, Planet. Space Sci., 27, 939, 1979b.

Allan, W., E. M. Poulter, and E. Nielsen, Pc5 pulsations associated with ring current proton drifts: STARE radar observations, Planet. Space Sci., 31, 1279, 1983.

Chisham, G., Giant pulsations: An explanation for their rarity and occurrence during geomagnetically quiet times, J. Geophys. Res., 101, 24,755, 1996.

Glassmeier, K.-H., Reply to the comment on Glassmeier et al. (1999) by I.R. Mann and G. Chisham, Ann. Geophysicae, In press, 1999.

Glassmeier, K.-H., S. Buchert, U. Motschmann, A. Korth, and A. Pedersen, Concerning the generation of geomagnetic giant pulsations by drift-bounce resonance ring current instabilities, Ann. Geophysicae, 17, 338, 1999.

Mann, I. R., and A. N. Wright, Finite lifetimes of ideal poloidal Alfvén waves, J. Geophys. Res., 100, 23,677, 1995.

Southwood, D. J., A general approach to low-frequency instability in the ring current plasma, J. Geophys. Res., 81, 3340, 1976.

Southwood, D. J., and M. G. Kivelson, Charged particle behaviour in low-frequency geomagnetic pulsations, 2, Graphical approach, J. Geophys. Res., 87, 1707, 1982.

Southwood, D. J., J. W. Dungey, and R. J. Etherington, Bounce resonant interactions between pulsations and trapped particles, Planet. Space Sci., 17, 349, 1969.

Takahashi, K., L. J. Zanetti, T. A. Potemra, and M. H. Acuna, A model for the harmonic of compressional Pc5 waves, Geophys. Res. Lett., 14, 363, 1987. 